\title{
A successful treatment of hypercalcemia with zoledronic acid in a 15-year-old boy with acute lymphoblastic leukemia
}

\author{
Hye-Jin Park, MD, \\ Eun-Jin Choi, MD, PhD, \\ Jin-Kyung Kim, MD, PhD
}

Department of Pediatrics, Catholic University of Daegu School of Medicine, Daegu, Korea
Received: 15 March, 2016

Revised: 18 April, 2016

Accepted: 24 May, 2016

Address for correspondence: Jin-Kyung Kim, MD, PhD

Department of Pediatrics, Catholic University of Daegu School of Medicine, 33 Duryugongwon-ro 17gil, Nam-gu, Daegu 42472, Korea

Tel: +82-53-650-4240

Fax: +82-53-621-4106

E-mail:kimjk@cu.ac.kr
Severe hypercalcemia in children is a rare medical emergency. We present a case of a 15-year-old boy with hypercalcemia (total calcium level, $14.2 \mathrm{mg} / \mathrm{dL}$ ) with a normal complete blood count, no circulating blasts in the peripheral blood film, and no other signs of acute lymphoblastic leukemia (ALL), including no signs of lymphadenopathy or hepatosplenomegaly. The hypercalcemia was successfully treated with zoledronic acid. As hypercalcemia can be the only presenting symptom of ALL in children, the diagnosis is often delayed. In children presenting with hypercalcemia, malignancies must be considered in the differential diagnosis.

Keywords: Hypercalcemia, Leukemia, Osteoclast activating factor

\section{Introduction}

The clinical presentation of hypercalcemia is often asymptomatic or vague. Although the signs and symptoms of hypercalcemia are similar regardless of the etiology, an increase in net calcium mobilization from the bones is generally the primary cause. In adulthood, primary hyperparathyroidism and malignant diseases are the most common causes of hypercalcemia ${ }^{1)}$. In children, hypercalcemia is less common, and the etiologies are diverse and age specific ${ }^{1,2)}$. In older children and adolescents, the main causes are primary hyperparathyroidism, prolonged immobilization, and malignancies ${ }^{2}$. Excessive vitamin D ingestion or release of endogenous 1,25-dihydroxyvitamin D from disease-activated macrophages can lead to hypercalcemia. The granulomatous diseases associated with extra-renal overproduction of vitamin D include sarcoidosis, tuberculosis, leprosy, disseminated candidiasis, and lymphoma. Other unusual causes such as medications associated with hypercalcemia include chronic excessive intake of vitamin A, thiazide diuretics, lithium carbonate, thyrotoxicosis, acute adrenal insufficiency, and insufficient phosphate supplementation in parenteral nutrition must be considered ${ }^{1.2)}$.

Hypercalcemia related to malignancies is observed frequently in some adult malignancies. However, hypercalcemia occurs in less than $1 \%$ of children with malignancies ${ }^{3,4)}$. The pediatric malignancies associated with hypercalcemia are rhabdomyosarcoma, neuroblastoma, hepatoblastoma, medulloblastoma, angiosarcoma, lymphoma, and leukemia. Most cases of hypercalcemia are associated with acute lymphoblastic leukemia (ALL) ${ }^{1-3)}$.

A LL, the most common malignancy in children and adolescents, usually presents with fever, pallor, bleeding, arthralgia, lymphadenopathy, and hepatosplenomegaly, but rarely presents with hypercalcemia. The incidence of hypercalcemia associated with leukemia seems to be only around $0.6 \%$ to $4.8 \%{ }^{5,6}$. Hypercalcemia was present in only 11 of 2,816 children with leukemia or lymphoma followed up at St. Jude Hospital between 1962 and 1991 ${ }^{3)}$. In another study, hypercalcemia was reported in only 22 children with ALL between 1990 and 2005 in $\operatorname{Japan}^{7)}$. Despite the small number of cases, most patients with childhood ALL who present with hypercalcemia at diagnosis share similar clinical characteristics such as nonspecific 
symptoms of hypercalcemia, lacking all classical features of malignancy, older age (second decade of life), and normal or low white blood cell counts with rare or absent circulating blasts in the peripheral blood film. Diagnosis is often delayed due to these nonspecific clinical characteristics ${ }^{3,5,7)}$.

We present the case of a 15 -year-old boy with severe hypercalcemia with a normal full blood count without circulating blasts, and no other signs of ALL, including no lymphadenopathy or hepatosplenomegaly. He was later diagnosed with ALL and his hypercalcemia was successfully treated with zoledronic acid.

\section{Case report}

A 15-year-old boy presented with pain in both hips and occasionally in the right thigh, and nausea, vomiting, anorexia, weight loss $(7 \mathrm{~kg})$ for a period of 2 months, and intermittent fever for 2 weeks prior to admission. Before referral to us, he made several visits to a local outpatient medical center with no definite diagnosis. On admission to our unit, he was lethargic and had hypertension (blood pressure, 150/100 mm $\mathrm{Hg}$ ), pulse rate of 84 beats/min, respiratory rate of 20 breaths/ min, and a body temperature of $37.3{ }^{\circ} \mathrm{C}$. Physical examination showed chronic illness, but no other signs, including no signs of lymphadenopathy or hepatosplenomegaly. His weight, height, and body mass index were $53 \mathrm{~kg}$ (25th percentile), $181.6 \mathrm{~cm}$ ( $>97$ th percentile), and $16.1 \mathrm{~kg} / \mathrm{m}^{2}$ ( $<3 \mathrm{rd}$ percentile), respectively. He was born at term with a birth weight of 3,500 g by uncomplicated spontaneous vaginal delivery and had no specific medical history. His family medical history was nonspecific.

Initial laboratory data revealed severe hypercalcemia with a serum calcium level of $14.2 \mathrm{mg} / \mathrm{dL}$, phosphorus level of 4.4 $\mathrm{mg} / \mathrm{dL}$, blood urea nitrogen level of $28.2 \mathrm{mg} / \mathrm{dL}$, and creatinine level of $1.3 \mathrm{mg} / \mathrm{dL}$. His serum intact-parathyroid hormone (PTH) level was low at $<2 \mathrm{pg} / \mathrm{mL}, 25$-hydroxyvitamin D level was normal at $35.5 \mathrm{ng} / \mathrm{mL}, 1,25$-dihydroxyvitamin D level was low at $4.73 \mathrm{pg} / \mathrm{ml}$, calcitonin level was low at $<2 \mathrm{pg} / \mathrm{mL}$, and PTH-related peptide level was normal at $1.2 \mathrm{pmol} / \mathrm{L}$. Additional laboratory findings are shown in Table 1.

Mildly increased parenchymal echogenicity was observed on a renal ultrasonography. Rradiography of the pelvis showed focal osteolytic lesions in the intertrochanteric area of right femur (Fig. 1). A coronal spin echo T1-weighted magnetic resonance image showed diffuse decreased marrow signal intensity in the vertebral column, pelvic bones, and both femurs, and several focal osteonecrotic areas in both iliac wings and the intertrochanteric area of the right femur (Fig. 2). There was mild and diffusely increased tracer uptake on both proximal humeri and femurs on a whole body bone scan with ${ }^{99 \mathrm{~m}} \mathrm{Tc}$ hydroxymethylene diphosphonate (Fig. 3).

The hypercalcemia was initially treated with intravenous isotonic saline and furosemide, with a very mild response. Before the underlying diagnosis was made, due to the persistent hypercalcemia, we decided to treat the patient with zole- dronic acid (4 mg, 1A) intravenous infusion for 30 minutes in $100 \mathrm{~mL}$ of isotonic saline. After a single dose of zoledronic acid, serum calcium levels progressively decreased and his serum creatinine level normalized within 48 hours. Adverse effects included fever $\left(38^{\circ} \mathrm{C}-39^{\circ} \mathrm{C}\right)$ for 2 days, myalgia, anemia (hemoglobin, $10.7 \mathrm{~g} / \mathrm{dL}$ ), thrombocytopenia (platelet count, $98,000 / \mu \mathrm{L})$, hypocalcemia $(6.7 \mathrm{mg} / \mathrm{dL})$, and hypophosphatemia $(1.5 \mathrm{mg} / \mathrm{dL})$, which developed over the following weeks despite supplementation with calcium (Fig. 4). Although peripheral blood smears showed a normal full blood count and no circulating blasts, the findings of hypercalcemia and the magnetic resonance imaging led to the suspicion of a hematological malignancy. A bone marrow examination was performed and the findings were also indicative of ALL, remission induction therapy was initiated. Currently, approximately 20 months after starting treatment, he remains in remission on maintenance chemotherapy. There has been no recurrence of hypercalcemia.

\section{Discussion}

It is helpful to consider the causes of hypercalcemia as either PTH-mediated or non-PTH mediated ${ }^{1)}$. Increased or nonsuppressed serum PTH level, no history of renal

Table 1. Laboratory test results on admission

\begin{tabular}{lcc}
\hline Variable & Results & Reference value \\
\hline Hemoglobin (g/dL) & 13.8 & $14.0-18.0$ \\
Hematocrit (\%) & 40.1 & $42-52$ \\
White blood cell count (/ML) & 8,000 & $5,000-10,000$ \\
Platelet count (/ $\mu \mathrm{L})$ & 203,000 & - \\
Aspartate transaminase (IU/L) & 17 & $15-40$ \\
Alanine transaminase ( IU/L) & 12 & $5-45$ \\
Lactate dehydrogenase (U/L) & 1,076 & $120-330$ \\
Alkaline phosphatase (U/L) & 158 & $30-130$ \\
Protein (g/dL) & 7.3 & $6.6-8.2$ \\
Albumin (g/dL) & 4.7 & $4-5.3$ \\
Cholesterol (mg/dL) & 121 & $<170$ \\
Uric acid (mg/dL) & 8.1 & $3-7.7$ \\
Calcium (mg/dL) & 14.2 & $8.2-10.2$ \\
Phosphorus, inorganic (mg/dL) & 4.4 & $2.7-4.7$ \\
lonized calcium (mmol/L) & 1.54 & $1.22-1.37$ \\
Blood urea nitrogen (mg/dL) & 28.2 & $7-18$ \\
Creatinine (mg/dL) & 1.3 & $0.5-1.2$ \\
iPTH (pg/mL) & $<2$ & $10-65$ \\
25(OH)D3 (ng/mL) & 35.5 & $30-100$ \\
1,25(OH)2 D3 (pg/mL) & 34.73 & $30-100$ \\
Calcitonin (pg/mL) & $<2$ & $<8.4$ \\
PTHrP (pmol/L) & 1.2 & $0-1.1$ \\
Spot urine-calcium/creatinine ratio & 0.83 & $<0.2$ \\
24-Hour urine calcium (mg/kg/day) & 11 & $<4$ \\
TRPi (\%) & 63 & $85-90$ \\
\hline iPTH, intact-parathyroid hormone; 25(OH)D3, 25-hydroxyvitamin \\
D; 1,25(OH)2D3,1,25-dihydroxyvitamin D; PTHrP, PTH-related \\
peptide;TRPi, tubular reabsorption of phosphate. & \\
& & \\
& &
\end{tabular}


failure, and not taking drugs or vitamin D suggests primary hyperparathyroidism $^{8}$. When there is a low PTH level (below $20 \mathrm{pg} / \mathrm{mL}$ ), PTH-related peptide (PTHrP) and vitamin D metabolites should be measured to assess for malignancy and vitamin D intoxication. If PTHrP and vitamin D metabolites are also low, stimulation of bone resorption and unrecognized calcium intake in the context of renal insufficiency should be considered $^{1,2,8)}$.

In the present case, based on the suppressed PTH, normal 25-hydroxyvitamin D, low 1,25-dihydroxyvitamin D, nonelevated serum phosphorous level, increased calcium/creatinine ratio from a random spot urine test, and decreased tubular reabsorption of phosphate, we assumed that the hypercalcemia was PTHrP mediated. However, the PTHrP level was not increased. There is a possibility that other bone resorption stimulating factors could be part of the pathogenic mechanisms

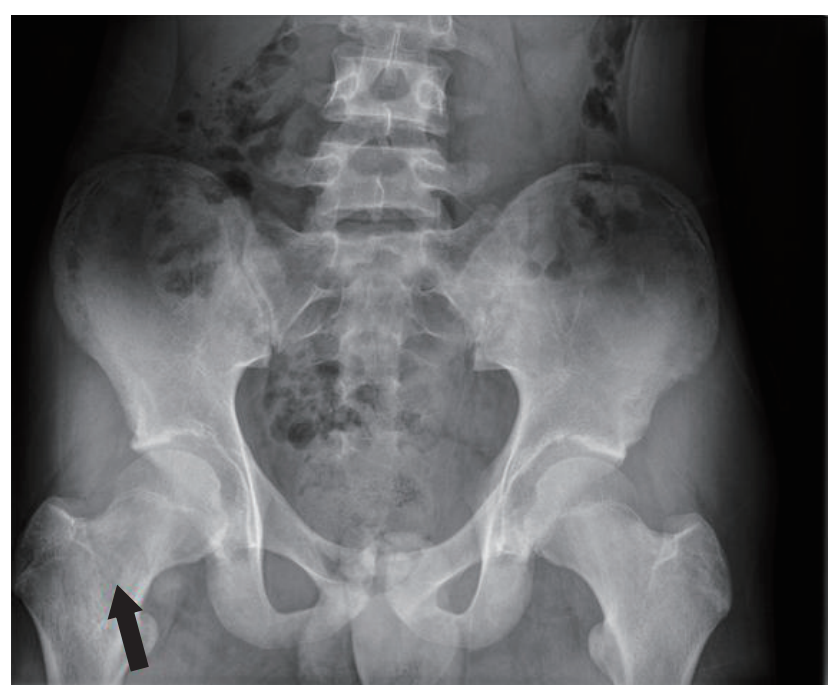

Fig. 1. Rradiography of the pelvis showed focal osteolytic lesions in the intertrochanteric area of right femur (black arrow). responsible for the hypercalcemia.

Hypercalcemia can occur in malignancy via 2 mechani$\mathrm{sms}^{1,2,5)}$. The first mechanism is associated with direct bone metastasis, with local release of various cytokines and other osteoclast-stimulating factors directly onto the surface of the bone in areas surrounding the malignant cells. The second mechanism is mediated by humoral factors released from tumor cells into the circulation. This mechanism accounts for up to approximately $80 \%$ of patients with hypercalcemia of malignancy ${ }^{9)}$. Parathyroid hormone-related peptide (PTHrP) is the most common cause ${ }^{5,6,9,10)}$. Under normal physiological conditions, PTHrP is secreted by various cells and participates in the development of tissues ${ }^{9,10)}$. In hypercalcemia of malignancy, PTHrP expression is enhanced ${ }^{10,11)}$. PTHrP and PTH are encoded by related genes that are part of a larger PTH gene family. PTHrP, structurally homologous with PTH, acts on the same type-1 PTH/PTHrP receptor. Therefore, their effects consistently overlap ${ }^{10)}$. Other bone resorption stimulating factors, such as tumor necrosis factor-alpha (TNF- $\alpha$ ), transforming growth factor-alpha, and interleukins have also been implicated ${ }^{9,11)}$. Rarely, malignant cells produce PTH ectopically $^{11)}$. 1,25-dihydroxyvitamin D induced hypercalcemia has also been seen in patients with lymphoma,11).

The cause of hypercalcemia in ALL may be related to the production of an osteoclast-activating factor by leukemic blasts. PTHrP is the most frequently involved mediator ${ }^{5,6)}$. Other cytokines, such as TNF- $\alpha$, interleukin-6, interleukin-2, prostaglandin $\mathrm{E}_{2}$ Level have also been implicated ${ }^{5,6,12-14)}$. PTH secretion by leukemic blasts has also been reported ${ }^{14)}$. In children with leukemia, hypercalcemia is more likely to occur at the time of initial diagnosis ${ }^{3)}$. It appears that in children, the presence of hypercalcemia does not affect their prognosis ${ }^{6}$.

Treatment of hypercalcemia depends on the primary cause. The urgency and aggressiveness of management is based on both the severity of calcium elevation and the presence of symptoms. Patients with severe hypercalcemia (serum calcium
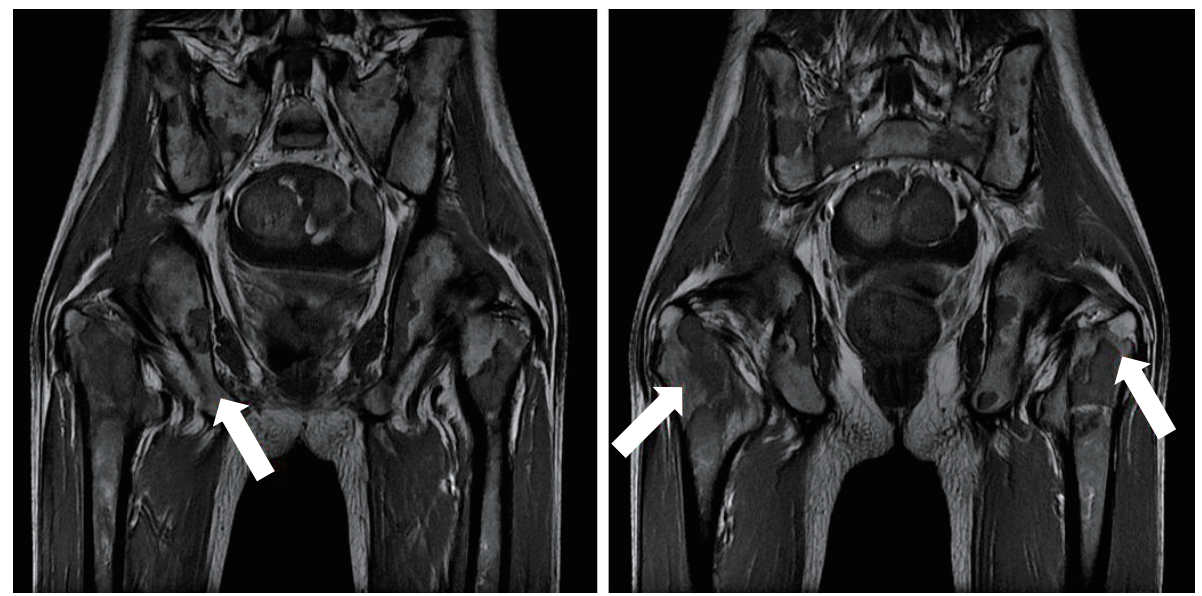

Fig. 2. A coronal spin echo T1-weighted magnetic resonance image showed diffuse decreased marrow signal intensity in the vertebral column, pelvic bones, and both femurs, and several focal osteonecrotic areas in both iliac wings and the intertrochanteric area of the right femur (white arrows). 


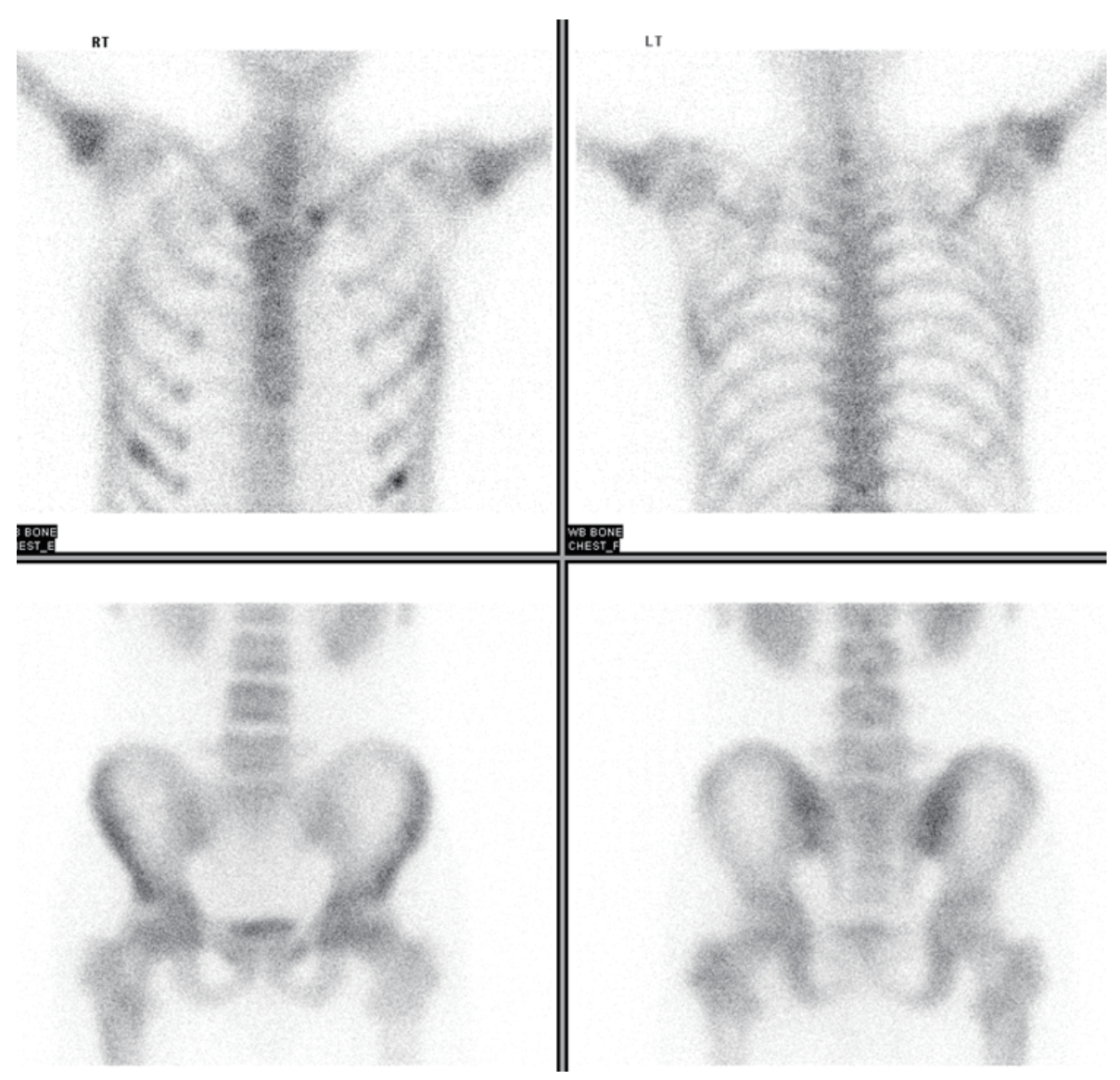

Fig. 3. There was mild and diffusely increased tracer uptake on both proximal humeri and femurs on a whole body bone scan with ${ }^{99 m} \mathrm{Tc}$ hydroxymethylene diphosphonate.

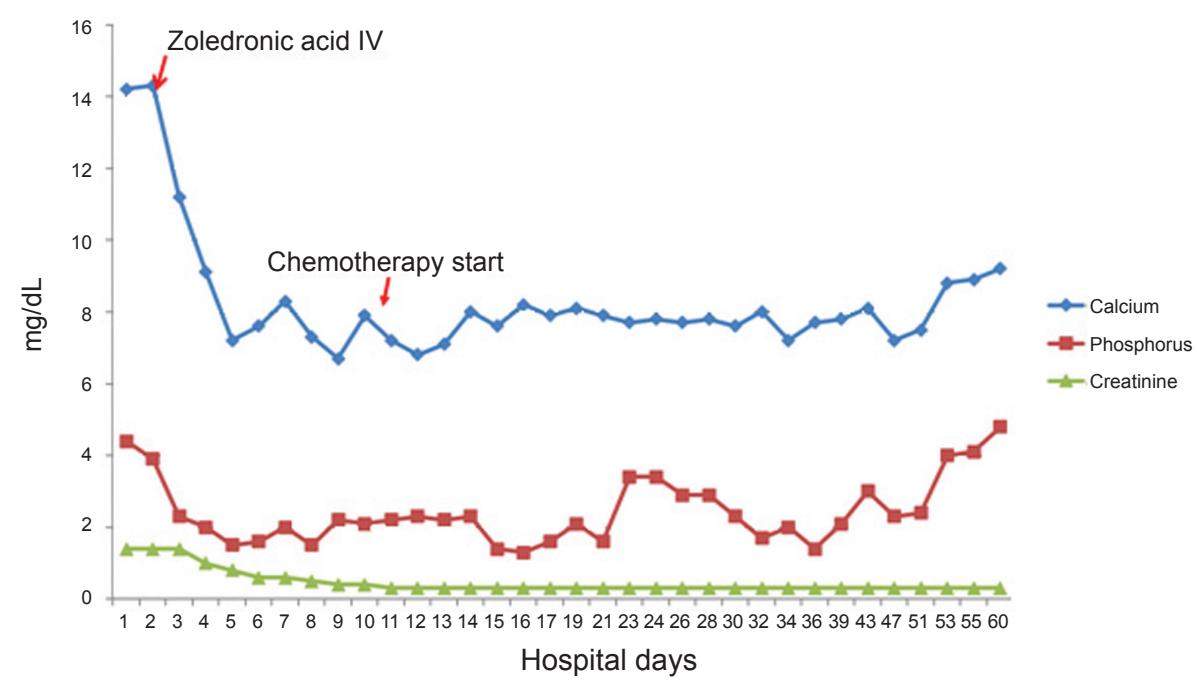

Fig. 4. Clinical courses; changes of serum calcium, phosphorus, creatinine level.

levels above $14 \mathrm{mg} / \mathrm{dL}$ ) are nearly always symptomatic and require prompt and comprehensive treatment. In moderate hypercalcemia (serum calcium levels between 12 and $14 \mathrm{mg} /$ $\mathrm{dL}$ ), treatment is based on symptoms and clinical judgment.
Hydration, enhanced renal calcium excretion by loop diuretics, and inhibiting bone resorption are the most important interventions ${ }^{11}$. Glucocorticoids inhibit 1a-hydroxylase and decrease 1,25-dihydroxyvitamin D production, and can be 
effective in hypercalcemia due to vitamin D intoxication or ectopic vitamin D production or hematologic malignancy ${ }^{11)}$. Calcitonin inhibits osteoclastic bone resorption, and, to a lesser extent, enhances renal calcium excretion. While a bisphosphonate provides a slow onset of action and a more sustained effect, the effect of calcitonin is prompt, short-lived, and can disappear after only a few days despite continued use $e^{5,11)}$ Therefore, calcitonin is most beneficial when combined with hydration and bisphosphonates ${ }^{11)}$. Bisphosphonates, such as pamidronate and zoledronic acid, are the preferred agents for the initial management of hypercalcemia caused by malignancy. Bisphosphonates adsorb to the surface of bone hydroxyapatite and act by blocking osteoclast-mediated bone resorption, rapidly lowering serum calcium levels, and the effects can last for weeks. Because bisphosphonates do not affect the renal tubular calcium reabsorption of PTHrP, patients with very high PTHrP levels may have a resistance to bisphosphonate treatment ${ }^{14)}$.

There are several reports that hypercalcemia resolved more rapidly in children treated with bisphosphonates compared with those who did not receive bisphosphonates ${ }^{1,2,45}$. However, cases refractory to bisphosphonates have also been reported. The 9-year-old patient was treated with pamidronate, but their serum calcium levels normalized only after the initiation of chemotherapy $^{14)}$. In pediatric patients, the potential adverse effects of bisphosphonates on the growing skeleton have been a main limitation to their use. Although experience in recent years has proven that bisphosphonate treatment is safe, strict followup of pediatric patients receiving long-term therapy is strongly recommended $^{15)}$.

Among the currently available bisphosphonates, zoledronic acid is considered more potent, effective, and convenient than pamidronate. Zoledronic acid at a dose of $4 \mathrm{mg}$ intravenous infusion over at least 15 minutes.is recommended for initial treatment of hypercalcemia of malignancy ${ }^{11,14}$. Adverse effects of zoledronic acid are common in the days immediately after administration, including acute phase reactions (flulike symptoms), hypocalcemia, hypophosphatemia, and less commonly, renal damage ${ }^{11,14)}$.

A short-term study of zoledronic acid in young patients ( 0.5 to 20 years) with bone disorders reported that acute adverse events were common, and occurred primarily after the first zoledronic acid infusion in patients with no previous bisphosphonate exposure, but these acute adverse events were mild and easily managed ${ }^{16)}$. Although reports on the use of zoledronic acid in leukemia patients are limited, few cases of successful treatment of hypercalcemia with zoledronic acid have been reported ${ }^{17-19)}$. For example, an 11.5-year-old boy with ALL and hypercalcemia (calcium, $13.8 \mathrm{mg} / \mathrm{dL}$ ) showed resolution of hypercalcemia within 48 hours after a single dose of zoledronic acid $(0.05 \mathrm{mg} / \mathrm{kg})^{17)}$. In addition, a 15 -year-old boy with ALL and hypercalcemia (calcium, $17.2 \mathrm{mg} / \mathrm{dL}$ ) showed resolution of hypercalcemia 24 hours after a single dose of zoledronic acid $(4 \mathrm{mg})^{18)}$. Also a 3 -year-old boy with ALL and hypercalcemia (calcium, $21.8 \mathrm{mg} / \mathrm{dL}$ ) showed resolution of hypercalcemia after continuous venovenous hemodiafiltration for 3 days and a single dose of zoledronic acid $(0.025 \mathrm{mg} / \mathrm{kg})^{19)}$. In Korea, a case of a 7-year-old girl ALL with hypercalcemia (calcium, 18.4 $\mathrm{mg} / \mathrm{dL}$ ) was reported in $2007^{13)}$. In this case, after massive fluid therapy with furosemide administration for 5 days while not used bisphosphonates, her calcium levels decreased to $12 \mathrm{mg} /$ $\mathrm{dL}$ and her calcium level finally normalized after initiation of chemotherapy. However, in our case, a 15 -year-old boy who received a single dose of zoledronic acid (4 mg), the serum calcium levels decreased rapidly and normalized within 48 hours.

Although the long-term efficacy and safety data are insufficient, we consider zoledronic acid to be safe and effective. To our knowledge, this is the first reported case of hypercalcemia in a patient with ALL who was successfully treated with zoledronic acid in Korea.

\section{Conflict of interest}

No potential conflict of interest relevant to this article was reported.

\section{References}

1. Davies JH, Shaw NJ. Investigation and management of hypercalcaemia in children. Arch Dis Child 2012;97:533-8.

2. Lietman SA, Germain-Lee EL, Levine MA. Hypercalcemia in children and adolescents. Curr Opin Pediatr 2010;22: 508-15.

3. McKay C, Furman WL. Hypercalcemia complicating childhood malignancies. Cancer 1993;72:256-60.

4. Kerdudo C, Aerts I, Fattet S, Chevret L, Pacquement H, Doz F, et al. Hypercalcemia and childhood cancer: a 7-year experience. J Pediatr Hematol Oncol 2005;27:23-7.

5. Trehan A, Cheetham T, Bailey S. Hypercalcemia in acute lymphoblastic leukemia: an overview. J Pediatr Hematol Oncol 2009;31:424-7.

6. Hibi S, Funaki H, Ochiai-Kanai R, Ikushima S, Todo S, Sawada T, et al. Hypercalcemia in children presenting with acute lymphoblastic leukemia. Int J Hematol 1997;66:3537.

7. Inukai $\mathrm{T}$, Hirose $\mathrm{K}$, Inaba $\mathrm{T}$, Kurosawa $\mathrm{H}$, Hama A, Inada $\mathrm{H}$, et al. Hypercalcemia in childhood acute lymphoblastic leukemia: frequent implication of parathyroid hormonerelated peptide and E2A-HLF from translocation 17;19. Leukemia 2007;21:288-96.

8. Eastell R, Brandi ML, Costa AG, D'Amour P, Shoback DM, Thakker RV. Diagnosis of asymptomatic primary hyperparathyroidism: proceedings of the Fourth International Workshop. J Clin Endocrinol Metab 2014;99:3570-9.

9. Mirrakhimov AE. Hypercalcemia of malignancy: an update on pathogenesis and management. N Am J Med Sci 2015;7:483-93

10. Wysolmerski JJ. Parathyroid hormone-related protein: an update. J Clin Endocrinol Metab 2012;97:2947-56.

11. Sternlicht H, Glezerman IG. Hypercalcemia of malignancy and new treatment options. Ther Clin Risk Manag 
2015;11:1779-88.

12. Niizuma H, Fujii K, Sato A, Fujiwara I, Takeyama J, Imaizumi M. PTHrP-independent hypercalcemia with increased proinflammatory cytokines and bone resorption in two children with CD19-negative precursor $\mathrm{B}$ acute lymphoblastic leukemia. Pediatr Blood Cancer 2007;49:990-3.

13. Lee YH, Lim YJ, Bae JJ, Kim JY, Shin JH. Hypercalcemia and extensive osteolytic lesion with increased plasma prostaglandin E2 level in a child with acute lymphoblastic leukemia. Korean J Hematol 2007;42:433-8.

14. Sargent JT, Smith OP. Haematological emergencies managing hypercalcaemia in adults and children with haematological disorders. Br J Haematol 2010;149:465-77.

15. Baroncelli GI, Bertelloni S. The use of bisphosphonates in pediatrics. Horm Res Paediatr 2014;82:290-302.

16. George S, Weber DR, Kaplan P, Hummel K, Monk HM,
Levine MA. Short-term safety of zoledronic acid in young patients with bone disorders: an extensive institutional experience. J Clin Endocrinol Metab 2015;100:4163-71.

17. Kolyva S, Efthymiadou A, Gkentzi D, Karana-Ginopoulou A, Varvarigou A. Hypercalcemia and osteolytic lesions as presenting symptoms of acute lymphoblastic leukemia in childhood. The use of zoledronic acid and review of the literature. J Pediatr Endocrinol Metab 2014;27:349-54.

18. Lokadasan R, Prem S, Koshy SM, Jayasudha AV. Hypercalcaemia with disseminated osteolytic lesions: a rare presentation of childhood acute lymphoblastic leukaemia. Ecancermedicalscience 2015;9:542.

19. Martins AL, Moniz M, Nunes PS, Abadesso C, Loureiro HC, Duarte X, et al. Severe hypercalcemia as a form of acute lymphoblastic leukemia presentation in children. Rev Bras Ter Intensiva 2015;27:402-5. 